\title{
LES ÉCRITS DE SUGER COMME SOURCE D'UNE ESTHÉTIQUE MÉDIÉVALE - UNE RELECTURE CRITIQUE
}

I.

Si l'on parle d'une esthétique médiévale, il y existe toujours un prototype éminent: la cathédrale gothique, et un témoin principal: la basilique de SaintDenis, l'abbaye royale franque, au nord de Paris à l'époque de Suger, ce dernier étant aussi qualifié de »créateur de l'art gothique «'. Parce que le chœur de l'église abbatiale consacré en 1144 est considéré comme le prototype des cathédrales gothiques, les écrits de Suger concernant l'abbaye de Saint-Denis sont interprétés comme des documents essentiels d'une "esthétique« médiévale. On y trouve certainement des descriptions longues et détaillées des consécrations et des transformations qu'ont connues les constructions dans la première moitié du XII ${ }^{e}$ siècle, et parmi ces transformations on relève surtout l'édification du »chœur de Suger« - défini par les »douze apôtres« et les »douze prophètes«, la double ligne des colonnes du déambulatoire ${ }^{2}$. L'image idéalisée de Suger comme père du style gothique et inventeur de l'»ars nova« s'identifie - mis à part les différents aspects archéologiques - à celle de la planification d'un prototype idéal supposé commun à tous les chevets de chœur, conception réalisée sur un »sol vierge«, libre de tout modèle et non influencée par les conditions extérieures ${ }^{3}$.

Voir Georges DuBY, Le temps des cathédrales, l'art et la société, 980-1420, Paris 1976, p. 175.

2 Cons 58, p. 349-351. - Les écrits de Suger sont cités d'après l'édition critique: Andreas SPEER, Günther BINDING, Abt Suger von Saint-Denis. Ausgewählte Schriften: Ordinatio, De administratione, De consecratione, Darmstadt 2000. Les abréviations suivantes sont utilisées: ord $=$ Ordinatio, cons $=$ De consecratione, $a d m=$ De administratione.

3 Le meilleur plan se trouve chez Jules FORMIGÉ, L'abbaye royale de Saint-Denis. Recherches nouvelles, Paris 1960, p. 108-109. En revanche, la figure la plus souvent utilisée par Dieter KIMPEL, Robert SUCKALE, Die gotische Architektur in Frankreich 1130-1270, Munich 1985, p. 89 (fig. 72) et par Dethard VON WINTERFELD, Gedanken zu Sugers Bau in St.-Denis, dans: Frank Neidhart STElGerwald (dir.), Martin Gosebruch zu Ehren. Festschrift anläßlich seines 65. Geburtstages, Munich 1984, p. 92-107, surtout p. 93, suggère par des retouches une régularité qui n'existe pas; il en est de même pour la voûte gothique. Une analyse détaillée des irrégularités de la partie orientale se trouve dans Jan 
Mais, cette »Meistererzählung ${ }^{4}$, cette relation maîtresse, est une invention moderne, une création d'Erwin Panofsky, qui s'appuie sur le mode interprétatif, se basant sur le modèle explicatif du néoplatonisme et de la métaphysique de la lumière. Avec cette approche, les constructions dans l'église abbatiale de Saint-Denis, décrites par Suger dans ses écrits concernant le sanctuaire et son couvent, sont interprétées dans leurs aspects de paradigmes du style naissant: on y voit alors la réalisation de la métaphysique de la lumière et du néoplatonisme en une architecture de lumière, où l'éclat divin et céleste brille dans la matérialité terrestre et permet l'ascension de l'intellect humain vers la connaissance de Dieu ${ }^{5}$. Dans l'introduction de l'édition procurée par Panofsky, le véritable point de départ de cette thèse est le portrait psychologique d'un abbé influent, original et actif, semblable à un mécène. Bruno Reudenbach a attiré l'attention sur la multiplicité des intentions contemporaines qui se trouvent dans l'image panofskyienne de Suger. Panofsky lui-même considère ce dernier comme le représentant d'une tradition spirituelle continue, fondée dans l'antiquité, survivant au Moyen Âge et existant encore aujourd'hui. Se référant à cette tradition menacée, le portrait de Suger construit par Panofsky - il l'a réalisé à l'occasion du $800^{\mathrm{e}}$ anniversaire de la consécration du chœur en 1944 - constituait une opposition humaniste à la barbarie du national-socialisme. La représentation stylisée de Suger en cette figure humaniste est donc basée sur son rattachement »à une tradition spirituelle qui reste toujours actuelle, dépassant les époques $\ll^{6}$.

VAN DER MEulen, Andreas SPEER, Die fränkische Königsabtei Saint-Denis. Ostanlage und Kultgeschichte, Darmstadt 1988, p. 9-22.

4 Cf. Arthur C. DaNTO, Kunst nach dem Ende der Kunst, Munich 1996, p. 20-21 (en anglais: Beyond the Brillo Box: The Visual Arts in Post-historical Perspective, New York 1992); sur ce sujet, voir mon article: Der erweiterte Kunstbegriff und das mittelalterliche "Kunst«-Verständnis, dans: Joseph Beuys und das Mittelalter (Katalog zur Ausstellung des Schnütgen-Museums vom 24. Januar bis zum 27. April 1997), Cologne 1997, p. 166175 , à la p. 167.

5 Erwin PANOFSKY, Abbot Suger on the Abbey Church of St.-Denis and Its Art Treasures (2nd ed. by Gerda PANOFSKY-SOERGEL), Princeton 1979, p. 18-26; cf. aussi Bruno ReudenBaCH, Panofsky und Suger von St. Denis, dans: ID. (dir.), Erwin Panofsky. Beiträge des Symposions Hamburg 1992, Berlin 1994 (Schriften des Warburg-Archivs im Kunstgeschichtlichen Seminar der Universität Hamburg, 3), p. 109-122, aux p. 113, 117 118 (traduction française: ID., Panofsky et Suger de Saint-Denis, dans: Revue germanique internationale 2 [1994], p. 137-150, aux p. 141-143, 145-147). Pour l'état des recherches concernant Suger, cf. Andreas SPEER, Abt Sugers Schriften zur fränkischen Königsabtei Saint-Denis, dans: SPEER, BINDING (voir n. 2), p. 13-66, surtout p. 13-18.

6 REUDENBACH (voir n. 5), p. 118 (trad. p. 147-148). 
II.

Il semble que Suger exprime précisément ces pensées dans ses vers, surtout dans ceux des portes principales dorées. Par le caractère anagogique de l'or, les portes montrent un chemin par lequel l'esprit engourdi peut, avec le soutien du »matériel«, s'élever vers le »véritable«, c'est-à-dire, vers la lumière vraie, où le Christ est la vraie porte ${ }^{7}$. Mais Erwin Panofsky déjà a remarqué dans ce passage devenu un locus classicus de l'interprétation de l'esthétique médiévale que l'on n'y trouve pas d'indice de la connaissance explicite de Denys par Suger si l'on respecte les critères stricts pour établir l'apparatus fontium d'une édition critique; on y trouve tout au plus des allusions plus générales, qui reflètent l'influence culturelle de Pseudo-Denys au XII ${ }^{\mathrm{e}}$ siècle. Ainsi, Panofsky cherchait des parallèles chez le deuxième traducteur - après Hilduin - du Corpus Dionysiacum, Jean Scot Érigène ${ }^{8}$. Mais cette argumentation, développée une nouvelle fois par Otto von Simson, peut seulement être qualifiée d'»hypothèse historique possible et plausible«, étant donné qu'il s'agit là d'une interprétation d'esthétique »où aucune épreuve historico-critique n'est donnée«?.

Les interprétations de Panofsky et de von Simson sont donc désormais l'objet de critiques ${ }^{10}$. En revanche, la recherche d'une idée esthétique directrice n'a pas encore perdu la fascination qu'elle engendrait. Actuellement, Hugues de Saint-Victor est encore une fois au centre des intérêts. Voilà par exemple les actes d'un colloque qui s'est tenu en automne 2000 à la Fondation Singer-Polignac sur le thème "L'abbé Suger, le manifeste gothique de SaintDenis et la pensée victorine«, maintenant édités par Dominique Poirel, qui a cherché de nouvelles indices pour une filiation depuis Denys jusqu'à Suger par Hugues ${ }^{11}$.

7 Adm 174, 775-783.

PANOFSKY (voir n. 5), p. 21-25.

9 Voir Werner BEIERWALTES, Negati affirmatio: Welt als Metapher. Zur Grundlegung einer mittelalterlichen Ästhetik durch Johannes Scotus Eriugena, dans: ID., Eriugena. Grundzüge seines Denken, Francfort-sur-le-Main 1994, p. 115-158, à la p. 158 . Voir aussi Otto VON SIMSON, Die gotische Kathedrale. Beiträge zu ihrer Entstehung und Bedeutung, Darmstadt 1968, p. 177-179 (en anglais: The Gothic Cathedral. Origins of Gothic Architecture and the Medieval Concept of Order, New York 1956, $2^{\mathrm{e}}$ éd. 1962); sur ce sujet, cf. Andreas SPEER, Stand und Methoden der Suger-Forschung, dans: Günther BINDING, Andreas SPEER (dir.), Abt Suger von Saint-Denis, De consecratione. Kommentierte Studienausgabe, Cologne 1995 (56. Veröffentlichung der Abteilung Architekturgeschichte des Kunsthistorischen Instituts der Universität zu Köln), p. 13-14.

${ }^{10}$ Sur ce sujet, voir SPEER (voir n. 5), p. 13-18.

1 Voir Dominique PoIREL, Symbolice et anagogice: l'école de Saint-Victor et la naissance du style gothique, dans: ID. (dir.), L'abbé Suger, le manifeste gothique de Saint-Denis et la pensée victorine (Actes du colloque à la Fondation Singer-Polignac, Paris, 21 novem- 
Otto von Simson considérait le commentaire d'Hugues sur l'écrit De caelesti hierarchia par le Pseudo-Denys comme le modèle idéal d'une méthode anagogique pour Suger ${ }^{12}$. Grover A. Zinn Jr. et Conrad Rudolph considèrent que Hugues est l'intermédiaire entre la pensée de Denys et celle de Suger ${ }^{13}$. Françoise Gasparri reprend cette thèse et relie le commentaire d'Hugues sur la "Hiérarchie céleste « à la réévaluation des arts mécaniques du Didascalicon et à des idées théologiques communes en christologie et en doctrine de Sacrement ${ }^{14}$. Pour sa part, Zinn se base sur les tituli du De administratione de Suger qui semblent correspondre à la théologie exprimée dans la préface du commentaire sur la »Hiérarchie céleste« de Denys par Hugues et sur la »Fenêtre d'anagogie«, dont les restes sont conservés aujourd'hui dans l'ancienne chapelle Saint-Pérégrin ${ }^{15}$. Quant à Rudolph, il considère que Hugues de SaintVictor est la tête pensante et productive du programme de construction à SaintDenis - nommée l'»artistic change - en se basant seulement sur le postulat d'une "communauté d'action " politique et théologique entre les abbayes de Saint-Denis et de Saint-Victor et entre leurs personnalités dominantes, Suger et Hugues ${ }^{16}$. Mais cette hypothèse - basée sur le concept méthodologique des "mental habits«, des »outillages mentaux" - n'est prouvée par aucun document, bien que la correspondance sugérienne soit très riche ${ }^{17}$. Cette hypothèse

bre 2000), Turnhout 2001 (Rencontres médiévales européennes, 1), p. 145-170, surtout p. $165-167$.

12 VON SIMSON (voir n. 9), p. 171-173. Cette hypothèse ne devient pas plus plausible si on suppose que les extraits du De hierarchia caelesti étaient lus in vigilia B. Dionysii comme lecture de table, comme Lindy GraNT, Abbot Suger of St-Denis. Church and State in Early Twelfth-Century France, Londres, New York 1998 (The Medieval World), p. 23-24 l'a supposé. Cf. aussi D. Philibert SCHMITZ, Les lectures de table à l'abbaye de SaintDenis vers la fin du moyen-âge, dans: Revue Bénédictine 42 (1930), p. 163-167, surtout p. 166.

${ }^{13}$ Grover A. ZINN, Jr., Suger, Theology and the Pseudo-Dionysian Tradition, dans: Paula Lieber GERSON (dir.), Abbot Suger and Saint-Denis. A Symposium, New York 1986, p. 33-40. Conrad RUDOLPH, Artistic Change at St-Denis. Abbot Suger's Program and the Early Twelfth-Century Controversy over Art, Princeton, New Jersey 1990.

${ }^{14}$ Françoise GASPARRI (éd.), Suger, Euvres, t. 1: Écrits sur la consécration de Saint-Denis; L'Cuvre administrative; Histoire de Louis VII, Paris 1996 (Les classiques de l'Histoire de France au Moyen Âge, 37), Introduction, p. XXXIII-XXXIX.

${ }^{15}$ ZINN (voir n. 13), p. 36, et Christoph MARKSCHIES, Gibt es eine "Theologie der gotischen Kathedraler? Nochmals: Suger von Saint-Denis und Sankt Dionys vom Areopag, Heidelberg 1995 (Abhandlungen der Heidelberger Akademie der Wissenschaften, philos.hist. Klasse, année 1995, 1. Abh.), p. 36 avec la n. 88 et les indications sur d'autres littératures sur la "fenêtre d'anagogie«. Le texte sur lequel se fonde Suger est adm 264-268; cf. sur ce sujet Susanne LINSCHEID-BURDICH, Beobachtungen zu Sugers Versinschriften in De administratione, dans: SPEER, BINDING (voir n. 2), p. 112-146, surtout p. 134-144.

${ }^{16}$ RUDOLPH (voir n. 13), p. 32-47 et p. 69-75, surtout p. 73; cf. aussi MARKSCHIES (voir n. 15), p. 37, et la recension de Joachim EHLERS, dans: Francia 21/1 (1995), p. 340-341.

${ }^{17}$ Parmi le corpus riche et varié des lettres de Suger, il n'y a pas un seul indice ou document d'une correspondance entre Suger et Hugues, dans Albert LECOY DE LA MARCHE, Euvres complètes de Suger, Paris 1867 (Société de l'Histoire de France. Publications, 139), 
se trouve donc au même status quaestionis que celles de Panofsky et de von Simson.

Évidemment, ces interprétations contiennent beaucoup de problèmes; je voudrais particulièrement souligner trois difficultés:

(i) concernant le caractère et le style des écrits de Suger ainsi que la question des sources;

(ii) concernant la relation entre textes et archéologie monumentale, et la possibilité et les limites d'une approche conjointe;

(iii) et finalement concernant les problèmes herméneutiques d'une idée esthétique directrice comme »esthétique de la lumière« ou »métaphysique de la lumière«.

III.

Commençons par un regard plus précis sur les trois textes dédiés à l'abbatiale et au monastère. Ces trois écrits - 1'Ordinatio, le De consecratione et le De administratione ${ }^{18}$ - font référence assurément à l'abbaye royale franque de Saint-Denis - l'église consacrée à saint Denis et les bâtiments conventuels mais aussi à Suger en tant qu'abbé, l'une dans l'entière complexité des tous les objets qui s'y trouvent, et l'autre dans toute l'étendue des tâches qui lui sont assignées. Par rapport aux fréquents problèmes de réception de cette œuvre, il faut d'abord préciser que l'homme politique Suger disparaît ici derrière le moine et l'abbé, qui, selon ses propres déclarations, mit toutes ses aspirations et toutes ses actions au service du couvent de Saint-Denis, où il fut d'abord placé comme oblat.

L'objet commun de ces textes est la campagne de travaux autour et à l'intérieur de l'église abbatiale, qui a abouti à trois consécrations. Ces dernières sont des indices importants pour la datation des trois écrits; la consécration du complexe occidental rénové a eu lieu le 9 juin 1140, la pose de la première pierre pour les travaux du complexe oriental, le 14 juillet 1140 et, finalement, la consécration de cette partie orientale, le 11 juin 1144. Les écrits font référence l'un à l'autre selon l'ordre chronologique de leur composition.

L'Ordinatio a été composée à la suite de la pose de la première pierre en présence du roi Louis VII, le 14 juillet 1140, devant l'ensemble du chapitre. Ce

Françoise GASPARRI (éd.), Suger, Euvres, t. 2: Lettres de Suger; Chartes de Suger; Vie de Suger par le moine Guillaume, Paris 2001 (Les classiques de l'Histoire de France au Moyen Âge, 41); voir aussi l'article de Jens Peter CLAUSEN dans ce volume (ci-dessous p. 109-116).

${ }^{18}$ Concernant la nouvelle édition critique, voir note 2. 
document, validé par quelques hommes d'Église puissants, personnellement obligés envers Suger, officialisa la grande œuvre de restauration portant sur les traditions cultuelles liées à Charles II le Chauve ainsi que sur la vie régulière. Cette restauration monastique et liturgique trouve sa justification dans la description finale de la pose de cette première pierre pour les transformations du complexe oriental. Cette cohérence, vérifiée par le dernier écrit $D e$ administratione - l'abbé lui-même renvoie explicitement à son ancien écrit ${ }^{19}$ - est importante pour comprendre la caractéristique des textes de Suger.

Le De administratione a été composé à la suite de la résolution du chapitre datée de la vingt-troisième année de l'abbatiat de Suger, l'année de la grande consécration du complexe oriental ${ }^{20}$. Dans le court prologue, il porte l'attention sur (i) l'acquisition (acquisitio) des nouveaux biens, la récupération (recuperatio) et l'augmentation (augmentatio) des anciens, perdus ou remis à neuf, (ii) la construction (constitutio ou institutio) des bâtiments, (iii) l'accumulation (repositio) et l'augmentation (augmentatio) des trésors d'or, d'argent, de pierres extrêmement précieuses et des chasubles (adm 1-2). L'augmentation des recettes y est intégrée dans le cadre d'une renovatio bénédictine en ce temps de défis intellectuels, sociaux et économiques, que Suger a documentés en détail. L'abbé visait là un but sacral: la transformation et le nouvel équipement de l'église abbatiale. Or, Suger témoigne de ce nouvel équipement en se référant à des documents contemporains ${ }^{21}$. Des indications tirées de ces sources se trouvent à la fois dans l'Ordinatio et dans le De consecratione et sont de nouveau reprises dans le De administratione. Pour sa part, le De consecratione, l'écrit de Suger qui est au centre des débats mentionnés au début, utilise textuellement un large extrait de l'Ordinatio concernant la pose de la première pierre du complexe oriental ${ }^{22}$. Il s'agit d'un passage qui concerne les rénovations des traditions cultuelles et les réformes de la vie monastique.

En résumé, on peut voir un contexte très précis pour les trois écrits mentionnés: le contexte monastique à Saint-Denis. Ce fait est confirmé par la transmission très restreinte de ces textes, qui ne sont évidemment produits que pour le monastère: l'Ordinatio, une charte conservée aux Archives nationales à Paris

${ }^{19}$ Adm 176, 791-792: quemadmodum in scripto consecrationis eiusdem superioris operis inuenitur.

${ }^{20}$ Pour la rédaction du De administratione, voir Martin PICKAVÉ, Zur Überlieferung der drei Schriften des Suger von Saint-Denis, dans SPEER, BINDING (voir n. 2), p. 147-159, à la p. 158.

${ }^{21}$ Sur Suger, voir Ludolf KUCHENBUCH, Ordnungsverhalten im grundherrlichen Schriftgut vom 9. bis zum 12. Jahrhundert, dans: Johannes FRIED (dir.), Dialektik und Rhetorik im frühen und hohen Mittelalter. Rezeption, Überlieferung und gesellschaftliche Wirkung antiker Gelehrsamkeit vornehmlich im 9. und 12. Jahrhundert, Munich 1997 (Schriften des Historischen Kollegs. Kolloquien, 27), p. 175-268, aux p. 253-257.

${ }^{22}$ Cons 50, 305-56, 341 = ord 38, 211-44, 248. 
(K $23 \mathrm{n}^{\circ}$ 5), le De consecratione, transmis dans deux codex mixtes, le premier du IX ${ }^{\mathrm{e}}$ jusqu'au XIII ${ }^{\mathrm{e}}$ siècle (aujourd'hui à la Bibliotheca Apostolica Vaticana, cod. Reg. lat. 571) et l'autre du XV $\mathrm{XV}^{\mathrm{e}}$ siècle (Paris, Bibliothèque de l'Arsenal, ms. 1030), qui contiennent plusieurs écrits variés concernant l'abbaye de Saint-Denis (par exemple les Gesta Dagoberti, une source très importante pour Suger), et finalement le De administratione, conservé dans un seul codex du $\mathrm{XIII}^{\mathrm{e}}$ siècle (Bibliothèque nationale de France, ms. lat. 13835). Nous avons aussi découvert une tradition indirecte dans une chronique anonyme rédigée à Saint-Denis au XIV $\mathrm{V}^{\mathrm{e}}$ siècle et transmise dans un codex du XV $\mathrm{XV}^{\mathrm{e}}$ siècle (Paris, Bibliothèque Mazarine, ms. 2017) ${ }^{23}$.

Les premières éditions de François Duchesne, Jean Mabillon, Michel Félibien dans sa fameuse "Histoire de l'Abbaye de Saint-Denys en France» de 1706 et aussi celle d'Albert Lecoy de la Marche, dont l'initiative revient à la Société de l'Histoire de France, renvoient à l'intérêt historique, qui commence au XVII ${ }^{\mathrm{e}}$ siècle en France ${ }^{24}$. Au début du XX ${ }^{\mathrm{e}}$ siècle, les écrits de Suger furent découverts par les historiens de l'art, en l'occurrence par Ernst Gall dans sa »Die Gotische Baukunst in Frankreich und Deutschland«, publiée en 1925, et finalement par Erwin Panofsky, qui eut une plus grande influence ${ }^{25}$.

Mais ce sont donc, tout à la fois, la réception sélective des textes de Suger et la réduction de leur sens à une seule cohérence qui ont contribué à la stabilisation des idées concernant la naissance du gothique à Saint-Denis: celles d'un Suger père du gothique, ou d'un Suger présenté comme père de la France, qui a clairement décrit les structures sociales et politiques du royaume capétien et qui, par l'idéologie, a présenté la conception de la royauté française ou l'aurait même créée ${ }^{26}$. En portant un regard attentif sur l'histoire de la recherche sur Saint-Denis, on perçoit clairement jusqu'à quel point la réception et l'interprétation d'un texte sont déterminées par les représentations directrices qui les motivent.

L'influence de ces idées directrices est aussi évidente dans les disciplines voisines comme, par exemple, l'histoire de la liturgie. Les deux études d'Anne Walters Robertson, concernant "The Service-Books of the Royal Abbey of Saint-Denis«, et d'Edward B. Foley, sur »The First Ordinary of the Royal Ab-

${ }^{23}$ Voir PICKAVÉ (voir n. 20), p. 145-152.

${ }^{24}$ Ibid. p. 152-154.

${ }^{25}$ Voir SPEER (voir n. 5), p. 15-16.

${ }^{26}$ Ibid. p. 17-18; cf. GRANT (voir n. 12), p. 3-21; Jean-François LEMARIGNIER, Le gouvernement royal aux premiers temps capétiens, 987-1108, Paris 1965; Michel BuR, Suger, abbé de Saint-Denis, régent de France, Paris 1991. Pour une relecture critique, voir Rolf GROSSE, Saint-Denis zwischen Adel und König. Die Zeit vor Suger (1053-1122), Stuttgart 2002 (Beihefte der Francia, 57), surtout p. 9-17 et p. 231-236. 
bey of St.-Denis in France (Bibliothèque Mazarine 526)«, adoptent le paradigme panofskyien sans le remettre en question ${ }^{27}$.

C'est pourquoi les vrais motifs de Suger et le caractère de ses écrits furent gravement ignorés pendant un certain temps, bien que Suger soit très clair. Dans l'introduction à son écrit sur la consécration, Suger mentionne lui-même l'idée directrice: proclamer tout haut ce bienfait unique et singulier entre tous de la largesse divine, rapporter aux donateurs les biens qu'ils en ont reçus, et porter à la connaissance des successeurs »la consécration glorieuse et digne de Dieu de cette sainte église et la translation très sacrée des très précieux martyrs, nos seigneurs et apôtres Denis, Rustique et Éleuthère et des autres saints, sur le patronage desquels nous nous fondons résolument«. Et Suger continue: "Nous avons exposé pour quelle raison, dans quel ordre, avec quelle solennité et avec quelles personnes cela fut accompli afin de rendre à la divine propitiation, suivant notre pouvoir, de dignes actions de grâces pour tel don et d'obtenir auprès de Dieu l'intercession favorable de nos saints protecteurs, tant pour les soins apportés à une telle ouvre que pour le récit d'une si grande solennité $\ll^{28}$. - Voilà un formidable accessus ad lectorem, très classique!

Cet «accessus« révèle le motif dominant, la liturgie, qui est aussi la clé pour traiter les trois problèmes mentionnés ci-dessus: (i) le caractère et le style des écrits de Suger et la question des sources; (ii) la relation entre textes et archéologie monumentale, et la possibilité d'une approche conjointe; (iii) la question d'une idée esthétique directrice.

${ }^{27}$ Anne Walters ROBERTSON, The Service-Books of the Royal Abbey of Saint-Denis. Images of Ritual and Music in the Middle Ages, Oxford 1991 (Oxford monographs on music); Edward B. FOLEY, The First Ordinary of the Royal Abbey of St.-Denis in France (Paris, Bibliothèque Mazarine 526), Fribourg 1990 (Spicilegium Friburgense, 32).

${ }^{28}$ Cons 7, 43-58: Iustificati igitur ex fide pace nostra interiori secundum apostolum pacem apud Deum habentes unum et inter multos singulare diuine largitatis beneficium more eorum, qui ad gratificandum impertita dona donatoribus suis ultro referunt, in medium proferentes gloriosam et Deo dignam sancte huius ecclesie consecrationem preciosissimorum martirum dominorum et apostolorum nostrorum Dyonisii, Rustici et Eleutherii et aliorum sanctorum, quorum prompto innitimur patrocinio, sacratissimam translationem ad successorum noticiam stilo assignare elaborauimus, qua de causa, quo ordine, quam sollempniter, quibus etiam personis ad ipsum actum sit, reponentes, ut et diuine propitiacioni pro tanto munere condignas pro posse nostro gratiarum acciones referamus et sanctorum protectorum nostrorum tam pro impensa tanti operis cura quam pro tante sollempnitatis adnotatione oportunam apud Deum optineamus intercessionem. (Traduction GASPARRI [voir n. 14], p. 7). 
IV.

La liturgie est l'élément déterminant pour comprendre le caractère et le contenu des trois écrits de Suger. Il est étonnant que cette dimension liturgique ait été ignorée depuis si longtemps. Cela ne s'applique pas seulement à l'ordo de la consécration de l'église selon le Pontificale Romanum du XII ${ }^{\mathrm{e}}$ siècle, soigneusement reconstruit par Michel Andrieu, particulièrement avec le traité $D e$ consecratione de Suger ${ }^{29}$, mais aussi à la juste compréhension des descriptions de la basilique et de ses transformations dans les textes de l'abbé.

Or, un regard porté sur les études concernant l'abbaye de Saint-Denis montre d'une manière exemplaire qu'il ne faut pas chercher dans les écrits de Suger des documents comparables aux descriptions d'édifices selon les modèles de l'histoire de l'architecture contemporaine, qui nous renseignent non seulement sur les motivations, mais aussi sur les formes concrètes. En particulier, les questions laissées ouvertes en archéologie ne se laissent pas résoudre par une exploitation simple des documents existants. Ainsi, ne s'étonnera-t-on pas de la déception d'Otto von Simson, l'historien de l'architecture, quand il découvre que »les problèmes techniques de construction « apparaissent, dans le De consecratione, "soit comme des difficultés décourageantes soit à l'occasion d'interventions célestes et miraculeuses ${ }^{30}$.

En réalité, la description la plus rigoureuse d'un bâtiment est celle de la vieille basilique attribuée à Dagobert $\mathrm{I}^{\mathrm{er}}$ (cons 9), où Suger utilise textuellement le passage correspondant des Gesta Dagoberti. Concernant l'usage de la terminologie architecturale (fundamentum, murus, columna, arcus, fenestra, etc.) et la dénomination des parties de l'édifice selon leurs fonctions spatiales traditionnelles (cripta, ala, oratorium, claustrum, etc.), Günther Binding a constaté que Suger ne se démarque pas de l'usage courant et qu'il semble manquer de précision en comparaison avec les rares autres exemples ${ }^{31}$. La valeur de ces descriptions apparaît seulement si l'on prend en compte l'intention descriptive, déterminée par le contexte liturgique. En effet, les différents pas-

${ }^{29}$ Michel ANDrIEU, Le Pontifical Romain au Moyen Âge, t. 1: Le Pontifical Romain du $\mathrm{XII}^{\mathrm{e}}$ siècle, Città del Vaticano 1938 (Studi e testi, 86). Voir sur ce sujet mes analyses dans: Abt Sugers Schriften (voir n. 5), p. 38-53.

${ }^{30}$ VON SIMSON (voir n. 9), p. 176.

${ }^{31}$ Pour le détail, voir Günther BNDING, Beiträge zum Architektur-Verständnis bei Abt Suger von Saint-Denis, dans: ID., SPEER (dir.), Mittelalterliches Kunsterleben nach Quellen

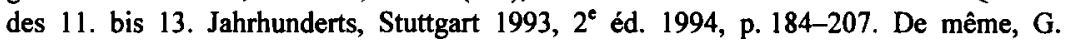
Boehm et H. Pfotenhauer constatent que les explications de Suger sur la construction de la cathédrale gothique pendant la première moitié du XII ${ }^{e}$ siècle ne contiennent pas de description de la construction, ni de son équipement comme d'un art spécifique; la description n'est pas non plus artistique ou expressive. Voir l'introduction par Gottfried BOEHM, Helmut PFOTENHAUER (dir.), Beschreibungskunst - Kunstbeschreibung, Munich 1995, p. 13. 
sages qui sont lus habituellement pour comprendre la forme des constructions apparaissent dans un certain contexte cultuel et liturgique, dont ils ne sont pas indépendants. Ils ne peuvent donc pas être compris dans le sens d'une simple description architecturale ${ }^{32}$.

C'est pourquoi il faut interpréter les descriptions de la construction en relation avec un contexte cultuel et liturgique, le modèle descriptif avec lequel Suger dirige le lecteur semblant être bien calculé. Dans la première partie du De administratione, Suger a guidé le lecteur à la manière d'un itinéraire imaginaire à travers l'abbaye. D'une façon similaire, l'ordre des objets de l'installation liturgique, des ornamenta, des thesauri et des vers et tituli peut être lu comme un système de renvois au moyen duquel Suger dirige le lecteur à travers son église, commençant à l'ouest ( $a d m$ 173-175) et poursuivant par le chœur ( $a d m$ 180-181). Ensuite il longe l'axe du centre liturgique du reliquaire (adm 197-200), passant par la croix d'or ( $a d m$ 201-213), jusqu'à l'autel matutinal (adm 240-242). À partir de ce lieu, il monte de nouveau dans le chœur vers les fenêtres de la chapelle axiale de la Vierge Marie, guidé par d'autres objets du mobilier comme la grande croix à côté du tombeau de Charles le Chauve et l'aigle d'or ( $\mathrm{adm} 262$ ). Puis on suit finalement l'arc de cercle nord (la fenêtre de Moïse, la fenêtre anagogique - $a d m$ 264-265). Ce modèle coïncide avec deux autres modèles de description: (a) concernant les événements liturgiques et particulièrement les processions et (b) à propos de l'ordre des autels et des vocables ${ }^{33}$.

Comme toutes les parties de la construction n'étaient pas encore terminées au moment de leur consécration, lorsqu'elles ont repris leur fonction liturgique (fait, qui n'était pas particulier au Moyen Âge), et comme Saint-Denis fut, pour cette raison, avant comme après, un grand chantier (en ce qui concerne les travaux de l'élargissement des transepts, par exemple, Suger n'en a fait que les fondations, "achevées par nous ou par ceux que Dieu a choisis ( $^{34}$ ), il est évident que c'est seulement grâce aux descriptions liturgiques que des parties de la construction disparates et souvent provisoires formaient une espace homogène et unique. Ainsi, le lecteur doit ressentir l'impression d'une unité déjà réalisée en un lieu, où - comme Suger l'exprime dans la prière finale de $D e$

32 Voir Andreas SPEER, Luculento ordine. Zum Verhältnis von Kirchweihliturgie und Baubeschreibung bei Abt Suger von Saint-Denis, dans: Nicolas BOCK e. a. (dir.), Kunst und Liturgie im Mittelalter. Akten des internationalen Kongresses der Bibliotheca Hertziana und des Nederlands Instituut te Rome, Rom 28.-30. September 1997, Munich 2000 (Römisches Jahrbuch der Bibliotheca Hertziana 33 [1999/2000], Beiheft), p. 19-37.

${ }^{33}$ SPEER (voir n. 5), p. 56-61. Voir aussi mon article: Suger et le chantier de Saint-Denis: pour une nouvelle lecture de ses écrits sur l'abbaye, dans: Textes et archéologie monumentale. Possibilités et limites d'une approche conjointe (Actes du Colloque international à Avignon, 30 novembre, $1^{\text {er }} \& 2$ décembre 2000) (sous presse).

${ }^{34} \mathrm{Adm}$ 186, 845-847: Sed quia iam inceptum est, in alarum extensione aut per nos aut per quos dominus elegerit, ipso auxiliante perficietur. 
consecratione - par le sacre et par la réception de l'eucharistie la plus sainte, le matériel et l'immatériel, le corporel et le spirituel, l'humain et le divin sont réunis dans une seule forme. Selon Suger l'église contemporaine se transforme par ces signes en un royaume céleste pour faire de nous et de la créature angélique, du ciel et de la terre, dans la toute-puissance et miséricorde divine, une seule république (unam rem publicam) ${ }^{35}$. Là, l'intention de Suger devient évidente: non pas décrire des parties d'église cloisonnées mais traiter de l'aménagement de la basilique comme d'un espace liturgique unique.

Quatre-vingts ans après la mort de Suger, les documents du XIII ${ }^{\mathrm{c}}$ siècle attestent, sous l'abbé Eudes, la reprise de travaux importants à Saint-Denis, que Pierre de Monterreux a finalement achevés ${ }^{36}$.

\section{V.}

Suger est pourtant conscient du résultat de ses rénovations. Il constate l'effet „de l'élargissement élégant et remarquable distribuant une couronne d'oratoires«, c'est-à-dire ce que l'on appelle le chœur de Suger, qui a traversé sans dommage les transformations du XIII ${ }^{\mathrm{e}}$ siècle. En revanche, la sentence - qui a été bien plus commentée - sur »la lumière merveilleuse et ininterrompue des vitraux qui resplendissaient quand elle les éclairait ${ }^{37}{ }^{3}$ 'atteste pas clairement l'existence d'une métaphysique de la lumière, mais tente plutôt de simplement décrire la luminosité du chœur pendant la journée, afin de rendre compte de la vision des moines qui recevaient ces rayons pendant les prières des heures,

${ }^{35}$ Cons 98, 614-621: ... qui sacramentali sanctissimi crismatis delibutione et sacratissime eucharistie susceptione materialia immaterialibus, corporalia spiritualibus, humana diuinis uniformiter concopulas, sacramentaliter reformas ad suum puriores principium, his et huiusmodi benedictionibus uisibilibus inuisibiliter restauras ecclesiam presentem, in regnum celeste mirabiliter transformas, ut, cum tradideris regnum Deo et patri, nos et angelicam creaturam, celum et terram unam rem publicam potenter et misericorditer efficias.

${ }^{36}$ VAN DER MEULEN, SPEER (voir n. 3), p. 300-301. Au sujet de l'aspect du chœur de Suger, toutes les spéculations restent ouvertes, voir Dieter KIMPEL, Robert SUCKALE, Die gotische Architektur in Frankreich 1130-1270, Munich 1985, p. 87.

${ }^{37}$ Cons 49, 301: ... illo urbano et approbato in circuitu oratoriorum incremento, quo tota clarissimarum uitrearum luce mirabili et continua interiorem perlustrante pulcritudinem eniteret. - Voir Andreas SPEER, Lwx mirabilis et continua. Anmerkungen zum Verhältnis von mittelalterlicher Lichtspekulation und gotischer Glaskunst (p. 89-94, surtout p. 9294) et H. WESTERMANN-ANGERHAUSEN, Glasmalerei und Himmelslicht - Metapher, Farbe, Stoff (p. 95-102, surtout p. 98-101), dans: Hiltrud WESTERMANN-ANGERHAUSEN (dir.), Himmelslicht. Europäische Glasmalerei im Jahrhundert des Kölner Dombaus (1248-1349) (Katalog zur gleichnamigen Ausstellung des Schnütgen-Museums vom 20.11.1998 bis 7.3.1999 in Köln), Cologne 1998. 
dans le cadre du service instauré par le roi Dagobert $\mathrm{I}^{\mathrm{er}}$ à Saint-Denis: la laus perennis $^{38}$. Il n'y a donc pas de raison de voir dans les idées platoniciennes des concepts concrets déterminant les programmes architecturaux, comme Sumner McKnight Crosby l'a admis pour vrai ${ }^{39}$, ni y identifier "l'élément moteur et créatif« qui servirait alors d'idée directrice ou d'wintention métaphysique«, comme l'ont supposé Erwin Panofsky, Otto Simson et Werner Beierwaltes ${ }^{40}$. Il faut en effet remarquer la nature des sources et des preuves: (i) des sources liturgiques et historiques comme l'Ordo Romanus et la Gesta Dagoberti, (ii) des poèmes religieux comme les carmina de Paulin de Nola, qui a organisé comme Suger à Saint-Denis - un agrandissement important de la basilique de Saint-Félix, ou de Venance Fortunat, et (iii) des modèles historiques comme la Narratio de consecratione ecclesiae Casinensis et la Chronica Monasterii Casinensis, la dernière attribuée à Léon d'Ostie. Ces sources et les prières, les chants liturgiques, les hymnes et lectures, qui forment une théologie vivante, donnent accès à des modèles d'explication plus proches de la réalité historique, comme les métaphores de la lumière - l'illumination de l'obscurité et la splendeur de la construction de l'église - que l'on trouve dans le rituel de la consécration lui-même et qui est en rapport avec la représentation de la liturgie terrestre comme image de la splendeur céleste ${ }^{41}$.

La question d'une esthétique médiévale a été tout particulièrement abordée au point d'intersection entre l'archéologie monumentale et l'approche des textes. Depuis ces points d'origine, Suger reste au centre du débat. Notre relecture de ses écrits sur l'abbaye permet un jugement nuancé. Ainsi, les travaux à Saint-Denis donnent un accès à l'expérience artistique (»Kunsterle-

${ }^{38}$ Hanns Peter NEUHEUSER, Die Kirchweihbeschreibungen von Saint-Denis und ihre Aussagefähigkeit für das Schönheitsempfinden des Abtes Suger, dans: BINDING, SPEER (voir n. 31), p. 116-183, surtout p. 154-157. Les documents les plus anciens sur l'installation d'un psallentium à côté des sépultures des saints patrons martyrs à Saint-Denis datent de la première moitié du VII ${ }^{e}$ siècle; voir VAN DER MEULEN, SPEER (voir n. 3), p. 137-138 avec n. 375-377.

${ }^{39}$ Sumner McKnight CrosBy, Crypt and Choir Plans at Saint-Denis, dans: Gesta 5 (1966), p. 4-8, en particulier p. 4-6; pour une analyse et critique détaillée, voir VAN DER MEULEN, SPEER (voir n. 3), p. 9-22, surtout p. 18-19. Une autre critique concerne la discussion de Peter Kidson avec Erwin Panofsky et Sumner McKnight Crosby sur les conditions géométriques du plan du chœur. Ces auteurs ont essayé de les reconstruire à partir de la théorie mathématique de Héron d'Alexandrie, cf. Peter KIDSON, Panofsky, Suger and St. Denis, dans: Journal of the Warburg and Courtauld Institute 50 (1987), p. 1-17, surtout p. $15-17$.

40 Voir BEIERWALTES (voir n. 9), p. 115.

${ }^{41}$ Voir le Pontificale Romanum XII s. (n. XVII), 30 (éd. ANDRIEU [voir n. 29], p. 182, 18 19): ... ut tenebras ab eo repellat et lumen infundat ... - Ibid. (n. XVII), 63 (éd. ANDRIEU, p. 191, 10-12): Horum igitur, domine, efflagitatus precibus, dignare hoc altare coelesti sanctificatione perfundere et benedicere. Assistant angeli claritatis et sancti spiritus illustratione praefulgeat. - Concernant les sources, voir: SPEER (voir n. 5), p. 31-53 et 65; LINSCHEID-BURDICH (voir n. 15), p. 112-146. 
ben $\ll)^{42}$, qui contraste avec la $»$ Meistererzählung« de la naissance du gothique. L'argumentation présentée ici est fondée sur une approche de cette expérience artistique, qui n'essaie pas de reconstruire, à travers les sources écrites, ses propres conceptions déterminées par des questions esthétiques modernes. Il s'agit d'un accès aux textes basé sur une méthode strictement historique, qui prend en considération l'horizon de la compréhension médiévale, ce qui implique la prise en compte du contexte des hommes, qui ont compris à leur manière les objets de leur époque que nous, nous définissons comme »œuvres d'art « ${ }^{43}$. Évidemment, les hommes ont toujours considéré et jugé divers faits en des temps différents. Mais, pour les comprendre selon leur époque, il faut comprendre leur mode de compréhension lui-même. Suger, par exemple, souligne constamment dans ses écrits la congruité et la cohérence de l'ancienne et de la nouvelle œuvre. Assurément, au terme de cette réflexion herméneutique, si nous jetons un regard rétrospectif sur le chantier de Saint-Denis au milieu du $\mathrm{XII}^{\mathrm{e}}$ siècle, Suger nous semble bien avoir ouvert la voie à une nouvelle conception architecturale. Mais nous ne pouvons pas confondre notre approche interprétative avec la compréhension et l'auto-interprétation de Suger luimême!

42 Sur ce concept, voir Andreas SPEER, Vom Verstehen mittelalterlicher Kunst, dans: BINDING, SPEER (voir n. 31), p. 13-52, surtout p. 36-40 et p. 49-52.

${ }^{43}$ Voir mes réflexions dans: Beyond Art and Beauty. In Search of the Object of Philosophical Aesthetics, dans: International Journal of Philosophical Studies 8 (2000), p. 73 88. 
